\title{
Rare Presentation of Pulmonary Echinococcosis
}

\author{
Natalya Kozlova, MD*; Mohamed Ramadan, MD; Christopher Mesa, MD; \\ Aijan Ukudeyeva, MD; Anna Shengelia, MD; Nadezhda Inragimova, MD; \\ Rajan Khanna, MD
}

Lincoln Medical and Mental Health Hospital, Bronx, NY, USA

\begin{abstract}
In this paper, we present a rare case of pulmonary cyst echinococcosis, in which the patient presented with no symptoms and was misdiagnosed as having pulmonary tuberculosis. Our case is a prime example of why echinococcosis should be part of the differential when dealing with an immigrant population. (International Journal of Biomedicine. 2017;7(2):138-140.)
\end{abstract}

Key Words: pulmonary cyst echinococcosis • tuberculosis • fine needle aspiration • albendazole

\section{Abbreviations}

AFB, acid-fast bacillus; CXR, chest X-ray; CT, computed tomography; FNA, fine needle aspiration; TB, tuberculosis

\section{Introduction}

Pulmonary cyst echinococcosis (PCE) is a parasitic infection caused by the tapeworm genus Echinococcus. Infections in the United States are unusual and are mostly among immigrants from endemic countries. Initially PCE is asymptomatic and incidentally found on a routine X-ray. Symptoms start to develop when cyst volume increases and applies pressure on surrounding tissues. The most common symptoms are cough, chest pain, dyspnea, and hemoptysis.

(1) Feared complications of cyst rupture are anaphylactic and septic shock, dissemination of cyst contents, along with empyema and pneumothorax. ${ }^{(1,2)}$

Diagnosis is usually established by a combination of the clinical and radiological presentation, where serology and cytomorphology are required for confirmation. In this paper, we present a rare case of PCE, in which the patient presented with no symptoms and was misdiagnosed as having pulmonary tuberculosis. After thorough evaluation in our hospital, patient was diagnosed with pulmonary cystic echinococcosis and received proper treatment.

*Corresponding author: Natalya Kozlova, MD. Lincoln Medical and Mental Health Hospital, Bronx, NY, USA. E-mail: natalya.kozlova77@gmail.com

\section{Case report}

A 19-year-old male walked into emergency department; (ED) with a self-reported TB history and requested treatment. The patient presented with no complaint, denied any history of cough, sputum production, shortness of breath, night sweating, or known TB contact. He reported he had migrated from Ecuador 1 month prior to the ED visit and was initially held in a detention facility while awaiting immigration clearance. While detained, he was diagnosed with TB and started on treatment with 4 medications. After his papers were cleared, he was advised to seek medical attention in any hospital at his final destination.

On initial presentation, a physical exam was unremarkable, CXR was significant for right lower lung mass measuring $3.4 \mathrm{~cm}$ (Fig. 1), CT chest (Fig. 2) was positive for well circumscribed RLL mass with a small peripheral area of cavitation or air pocket and no mediastinal lymphadenopathy, and laboratory revealed elevated transminases. Patient was placed on airborne isolation. Quantiferon TB Gold and AFB sputum and cultures were sent and are pending. His detention facility was contacted for additional collateral tests. Their investigation revealed similar CXR findings and a positive purified protein derivate test; however, they reported 3 negative AFBs. At this that time, he was started on active 
TB treatment. Our differential diagnosis remained broad, including hamartoma, granuloma, TB, and neoplastic process.

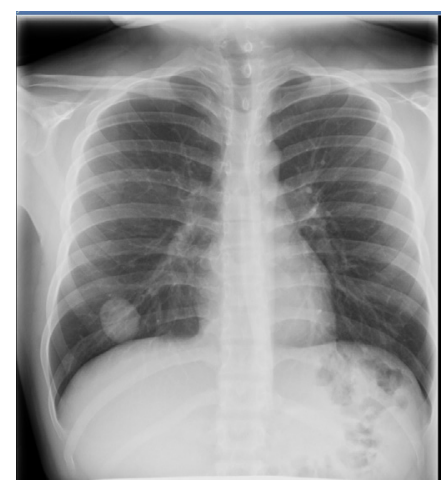

Fig. 1. Chest X-ray

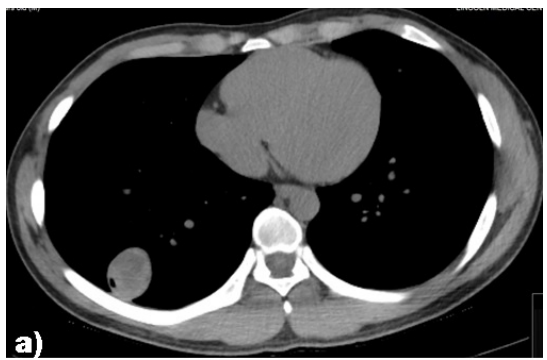

Fig. 2. Chest CT scan
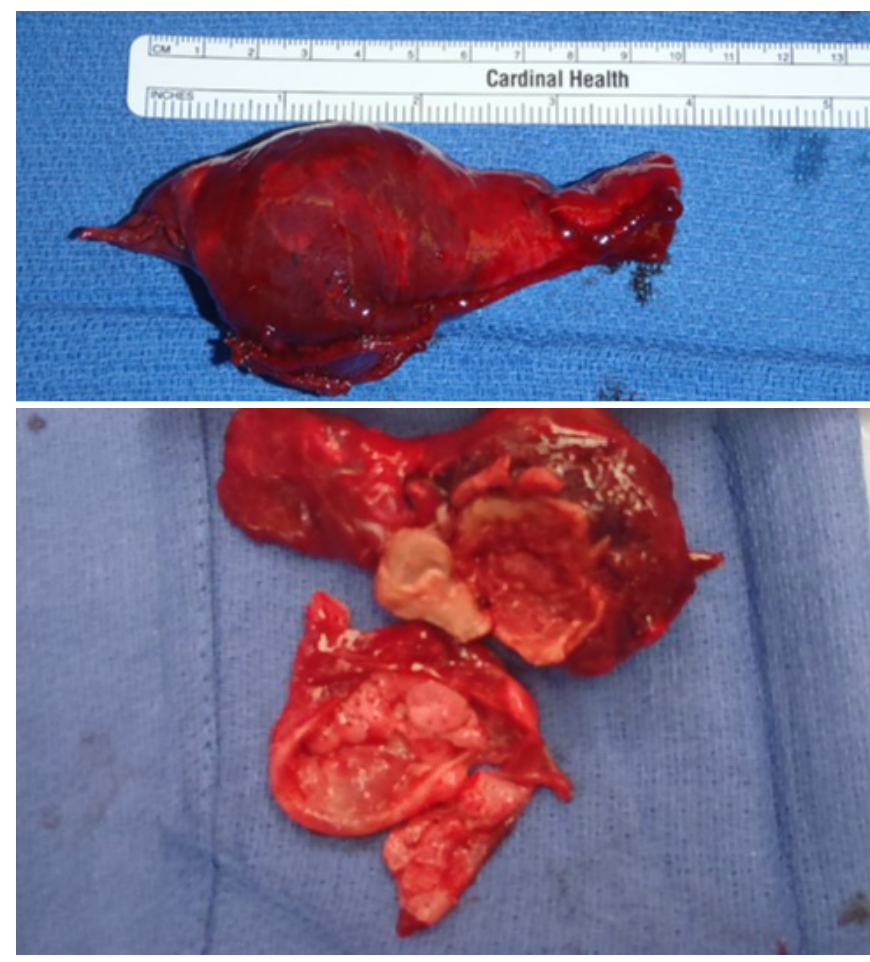

Fig. 3. Hydatid cyst removed during the surgery

Taking into account lack of symptoms, no history of TB contact, and low probability of $\mathrm{TB}$ on radiological images, active TB treatment was not re-initiated. By this time, the results of Quantiferon and 3 AFBs were negative. The decision was made to proceed with CT-guided FNA. An initial quick pathology read revealed particles of parasites, with a high suspicion of echinococcal cyst. Further work-up didn't find any features consistent with echinococcosis. No evidence of echinococcosis was found on CT abdomen or on brain. The final pathology report confirmed particles of echinococcal cysts. The patient underwent an open right thoracotomy with large wedge resection of the lung and was started on treatment with albendazole (Fig.3). Patient was discharged home in good condition and came for follow-up for suture removal, as well as parasite clinic for completion of albendazole therapy and had no complaints.

\section{Case discussion}

Echinococcus granulosus is a zoonotic parasitic infection caused by the larva of the dog tapeworm, and is known to cause PCE. ${ }^{(1)}$ It is of particular concern in Asia, South America, the Middle East and Africa, where its prevalence is close to $15 \% .^{(3,4)}$ Within the United States, PCE is quite unusual and mostly seen within immigrants from endemic countries. In an immigrant patient with a suspicious pulmonary lesion, PCE should be within the differential diagnosis. Raising awareness among physicians about PCE would decrease the associated disease burden.

Initially, PCE is asymptomatic and is often an incidental finding on routine X-rays. Symptoms begin to develop as the cyst enlarges and starts to apply pressure on surrounding structures. The most common symptoms are cough, chest pain, dyspnea, and hemoptysis. ${ }^{(1)} \mathrm{PCE}$ is most often diagnosed by a combination of clinical and radiological findings, which is then confirmed by serology and cytomorphology. Radiological diagnosis begins with CXR, which will show a typical well-defined cystic lesion. Unruptured cysts are often indistinguishable from a variety of other pulmonary lesions. ${ }^{(2,5)}$ As the cyst enlarges, it will erode neighboring bronchioles producing air accumulation between cyst layers. This results in the characteristic "meniscus," "onion peel," or "water-lily" signs seen on radiographic imaging. While CT is not needed to establish diagnosis, CT will reveal a more detailed picture of the cyst and of surrounding structures. In addition, abdominal ultrasound should be part of the routine work-up as $20 \%$ of PCE cases have concomitant liver involvement. ${ }^{(2)}$ Diagnostic percutaneous aspiration of the lung cyst is considered controversial due to possible cyst rupture, dissemination of cyst content, and anaphylaxis. Treatment of PCE is surgical removal with concurrent chemotherapy. The drug of choice is albendazole.

In our literature review of past publications, Echinococcosis is most often confused with lung malignancy. ${ }^{(6-8)}$ There are several cases of an atypical presentation, in which radiographic features on CXR and CT are suggestive of lung malignancy. FNA and bronchoscopy were inconclusive and diagnosis of PCE was established at the time of surgical removal.

\section{Conclusion}

Diseases once rare within the United States are now found to be more prevalent due to the increase in immigration. 
Because of this change, physicians should broaden their scope when considering their differential of infectious diseases. Our case is a prime example of why echinococcosis should be part of the differential when dealing with an immigrant population.

\section{Competing interests}

The authors declare that they have no competing interests.

\section{References}

1. Santivanez S, Garcia HG. Pulmonary cystic echinococcosis. Curr Opin Pulm Med. 2010; 16(3):257-61. doi: 10.1097/MCP.0b013e3283386282.

2. Arinc S, Kosif A, Ertugrul M, Arpag H, Alpay L, Unal O, et al. Evaluation of pulmonary hydatid cyst cases. Int J Surg. 2009;7(3):192-5. doi: 10.1016/j.ijsu.2008.11.003.

3. Moro P, Schantz PM. Cystic echinococcosis in the Americas. Parasitol Int. 2006;55 Suppl:S181-6.
4. Wang GZ, Feng XH, Chu XD, Er XD, Ar MN, Wen H. Epidemiological study on human echinococcosis in Hobukesar Mongolian autonomous county of Xinjiang. Chin J Endemiol. 2009;28:214.

5. Turgut AT, Altinok T, Topcu S, Kosar U. Local complications of hydatid disease involving thoracic cavity: imaging findings. Eur J Radiol. 2009;70(1):49-56. doi: 10.1016/j.ejrad.2008.01.002.

6. Kilinç O, Döskaya M, Sakar A, Yorgancioğlu A, Halilçolar H, Caner A, Gürüz Y.Three atypical pulmonary hydatidosis lesions mimicking bronchial cancer from Turkey. New Microbiol. 2009;32(2), 229-33.

7. Cobanoglu U, Asker S, Mergan D, Sayir F, Bilici S, Melek M. Diagnostic Dilemma in Hydatid Cyst: Tumor-Mimicking Hydatid Cysts. Turk Thorac J 2015;16:180-4.

8. Paksoy N, Özer D,Tuneli IO. Diagnosis of pulmonary hydatid disease presenting with solid nodule and mimicking malignancy by fine needle aspiration cytology. Cytojournal. 2012;9:13. doi: 10.4103/1742-6413.95832. 